\title{
A case of small hepatocellular carcinoma with an extensive lymph node metastasis at diagnosis
}

\author{
Young Mi Hong', Ki Tae Yoon', Mong Cho', Jeong Heo², Hyun Young Woo², and Won Lim² \\ 'Department of Internal Medicine, Pusan National University Yangsan Hospital, Pusan National University School of Medicine, Yangsan; \\ ${ }^{2}$ Department of Internal Medicine, Pusan National University Hospital, Pusan National University School of Medicine, Busan, Korea
}

\section{To the Editor,}

A 41-year-old man was admitted to our hospital for investigation of a liver mass with extensive lymph node (LN) enlargement that was detected during follow-up abdominal computed tomography (CT) for chronic viral hepatitis $B$. Laboratory investigations showed a serum alanine aminotransferase level was 31 IU/L (normal range 10-40 IU/L), the aspartate aminotransferase level was $23 \mathrm{IU} / \mathrm{L}$ (normal range 6-40 IU/L) and the $\gamma$-glutamyltransferase level was $44 \mathrm{IU} / \mathrm{L}$ (normal range 5-63 IU/L). Alkaline phosphatase, total bilirubin and albumin levels were all within normal limits. The patient had an elevated serum $\alpha$-fetoprotein level of $3580 \mathrm{ng} / \mathrm{mL}$ (normal range $\leq 10 \mathrm{ng} / \mathrm{mL}$ ) and a PIVKA-II level of $489 \mathrm{mAU} / \mathrm{mL}$ (normal range $0-40 \mathrm{mAU} / \mathrm{mL}$ ); moreover, the carcinoembryonic antigen level was high level of $31.1 \mathrm{ng} / \mathrm{mL}$ (normal range 0-5 ng/ $\mathrm{mL})$. Serum hepatitis e antigen was negative and HBV PCR DNA level was $156 \mathrm{IU} / \mathrm{mL}$. Abdominal CT showed hypodense mass, 2 $\mathrm{cm}$, with necrosis at the medial portion, at the liver S7. Adding to this, CT demonstrated multiple conglomerated LN enlargement at the celiac axis, superior mesenteric artery (SMA), and para-aortic area (Fig. 1). In addition to CT, magnetic resonance imaging (MRI) also showed $2 \mathrm{~cm}$ sized mass with necrosis at the medial portion, at the liver S7 and extensive LN enlargement. And FDG-PET/CT and chest $\mathrm{CT}$ were performed to evaluation of extrahepatic metas- tasis. FDG-PET/CT showed isometaoblic mass, S7 of the liver with extensive LN metastasis at neck, axilla, mediastinum, and retroperitoneum (Fig. 1). Based on these findings, we could suspected hepatic lymphoma or combined hepatocellular and lymphoma and liver biopsy was performed for confirmation of liver mass. However, because of no evidence of malignancy on histologic findings of liver mass, cervical excisional LN biopsy was conducted. Histologic findings revealed metastatic hepatocellular carcinoma (HCC) and we confirmed small hepatocellular carcinoma with extensive LN metastasis (Fig. 2). Base upon the diagnosis of HCC metastasis to the LN, we started therapy with sorafenib. Two months later after sorafenib administration, an abdomen $\mathrm{CT}$ revealed new multiple hypodense masses with central necrosis at the liver and unfortunately, the patient died 3.5 months after the detection of the liver mass.

The rate of extrahepatic metastases from hepatocellular carcinoma (HCC) is relatively lower than that in other hepatic cancers. ${ }^{1}$ Lymph node (LN) metastasis is observed in only $1.7 \%$ of patients undergoing surgery, but has been frequently observed in $30.7 \%$ of autopsies. ${ }^{2}$ It has been reported that small HCC, less than $2 \mathrm{~cm}$ in diameter, constitute approximately $15.6 \%$ of resectable HCC. $^{3}$ Moreover, as a follow up of a high risk group of HCC using serum AFP and abdominal ultrasound is increased, early detection of resectable small HCC is increased so that in the case of small HCC,

\section{Abbreviations:}

LN, lymph node; HCC, hepatocellular carcinoma; $C T$, computed tomography

\section{Corresponding author : Ki Tae Yoon}

Department of Internal Medicine, Pusan National University Yangsan Hospital, Beomeo-ri, Mulgeum-eup, Yangsan 626-770, Korea

Tel: +82-55-360-2362, Fax: +82-55-360-1737

E-mail:ktyoon@pusan.ac.kr 
Young Mi Hong, et al.

Small HCC with an extensive lymph node metastasis
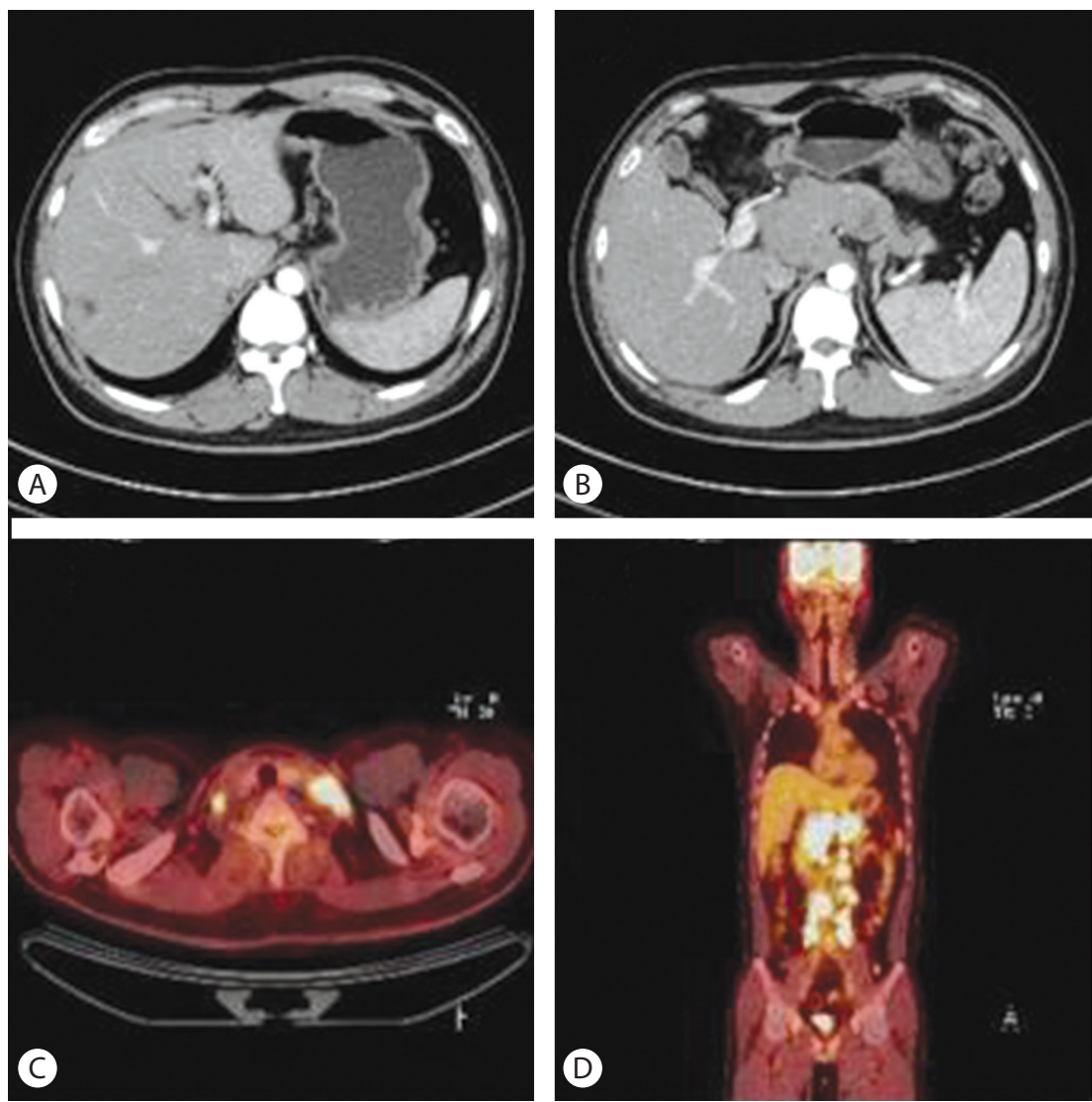

Figure 1. Abdomen computed tomography showed ( $A$ ) hypodense mass, in $2.5 \mathrm{~cm}$ diameter in arterial phase and (B) multiple conglomerated LNs enlargement at the celiac axis, SMA, and paraaortic area. FDG-PET/CT showed (C) increased uptake of multiple LNs such as both supraclavicular LNs and (D) multiple intraabdominal LNs (celiac axis, retropancreatic, right retrocaval, portocaval, aortocaval, paraaortic, common iliac bifurcation, both common iliac LN).
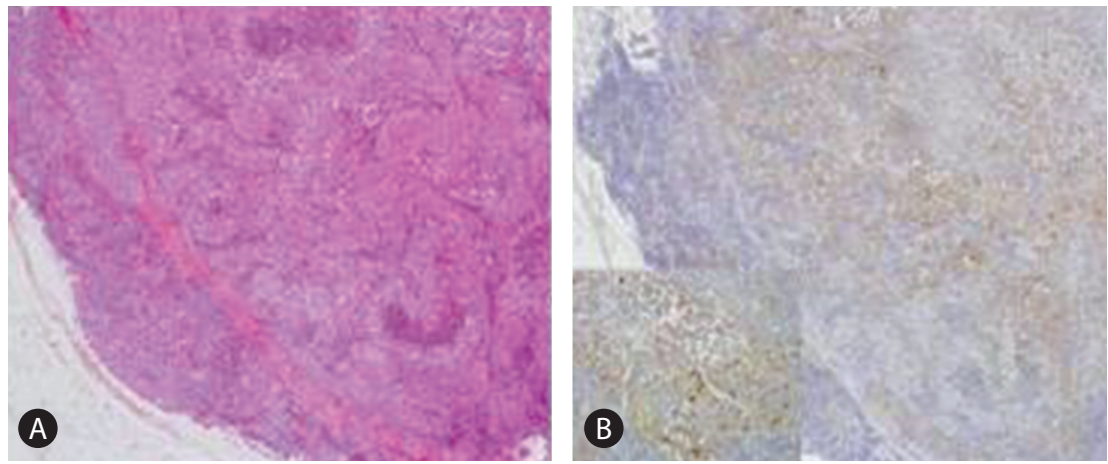

Figure 2. Microscopic findings of excisioned neck LN shows that (A) lymph node is replaced by tumor cell nests consisting of thick trabeculae with central tumor necrosis (H\&E staining, $\times 40$ ). (B) some tumor cells show positive reaction to HSA immunohistochemical staining. (observed under the high-power view (x200) in a box).

less than $2 \mathrm{~cm}$ in diameter, is showing more than $50 \%$ of a 5 -year survival rate. ${ }^{4}$

Because LN metastasis of small HCC are very rare and good prognosis, the clinical significance has not well investigated and the relationship between the type of a primary HCC lesion and type of accompanying lymph node metastasis has not been documented. There is one study to investigate the relationship between the type of a primary HCC and the type of accompanying lymph node metastasis. Although all of the primary HCC lesions are not small HCC, they suggested that we need to consider that the type of the primary lesion is related to the pattern of lymph node metastasis and systemic exploration for lymph node metastasis is important, even for patients with small nodular type $\mathrm{HCC}^{5}$

Although diagnosis of small HCC has been increasing, there are lack of studies to investigate small HCC characteristics and prognosis. Therefore, further investigation should be needed to identify small HCC showing poor prognosis. 


\section{Conflicts of Interest}

No potential conflict of interest relevant to this article was reported.

\section{REFERENCES}

1. Uehara K, Hasegawa H, Ogiso S, Sakamoto E, Ohira S, Igami T, et al. Skip lymph node metastases from a small hepatocellular carcinoma with difficulty in preoperative diagnosis. J Gastroenterol Hepatol 2003;18:345-349.
2. Liver Cancer Study Group of Japan. Survey and follow-up study of primary liver cancer in Japan: Report 17. Acta Hepatol Jpn 2007;48:117-140.

3. Liver Cancer Study Group of Japan. Survey and follow-up study of primary liver cancer in Japan: Report 12. Acta Hepatol Jpn 1997;38:317-330.

4. Nagashima I, Hamada C, Naruse K, Osada T, Nagao T, Kawano N, et al. Surgical resection for small hepatocellular carcinoma. Surgery 1996;119:40-45.

5. Abe T, Furuse J, Yoshino M, Kinoshita $T$, Konishi M, Inoue $K$, et al. Clinical characteristics of hepatocellular carcinoma with an extensive lymph node metastasis at diagnosis. Am J Clin Oncol 2002;25:318-323. 